\title{
Fundamental Irreversibility and the Concept of Events
}

\author{
Rudolf Haag \\ II. Institut für Theoretische Physik, Universität Hamburg, D-2000 Hamburg 50, \\ Federal Republic of Germany
}

Dedicated to Res Jost and Arthur Wightman, friends for many years and appreciators of unorthodox thought

\begin{abstract}
It is proposed that the transmutation from possibilities to facts should be introduced as an essential element in fundamental theory. This has no bearing on TCP-invariance. If indeterminism is accepted it leads to a picture of an evolving history formed by individual events and causal ties. In the low density regime it can be compared with the treatment of multiple collisions in quantum field theory.
\end{abstract}

\section{Introduction}

It is difficult and perhaps still somewhat controversial to summarize the tenets of quantum physics. Indeterminacy is one aspect which is easily understood and accepted by most. It is the recognition that the "laws of nature" do not allow the prediction of future development with certainty even if optimal knowledge of the past were available. The problem of "reality" touches a deeper level, a central nerve of physics and it has troubled many. Using the standard language and concepts developed during the early discussions by Bohr, Heisenberg, Dirac, von Neumann we must divide the universe into two parts: the "observed system" $\Sigma$ and the observer with his equipment $M$. The singling out of a system $\Sigma$ constitutes a (to some degree arbitrary) mental decision on the side of the observer but it was emphasized by Heisenberg that, while the cut can be shifted to some extent it cannot be avoided. The "system" cannot be the universe as a whole. The two parts, $\Sigma$ and $M$ have different aspects. On the side of $M$ there is the free will of the observer to plan an experiment and consciousness of results. A reduction of free will and consciousness to physical laws is not attempted. Furthermore, on the side of $M$, there is the need to describe the instruments and the findings in common language (the language of "classical physics" in the formulation of Bohr). Within this frame the task of the theory is to specify the general properties of possible systems (mass, spin, charges of particles, structure of matter...) and to predict the probability for finding a particular measuring result from the available knowledge about the past. 
The standard language places the observer in a central position. According to common language an observer is a human being; at least he has to be equipped with a mind. The theory refrains from talking about real events in $\Sigma$ unless they are observed phenomena. This was Schrödinger's criticism which he wanted to illustrate with the parabola of the cat who is neither dead nor alive. The notion of a "state" of $\Sigma$ concerns only the potentiality of producing a phenomenon by interacting with $M$ and this means that the event either reaches the consciousness of mind or that it becomes a macroscopically recorded, classically describable feature of the material parts included on the side of $M$. If one focuses on the former one might say that the ultimate position to which the cut may be moved is between the material world and the mind. This would, however, ban the notion of real events and history from the material world. The alternative is to use different parts of physics for the description of the material parts of $M$ as opposed to $\Sigma$. Then one must explain the recording, the transmutation from the microscopic world to a phenomenon in classical physics as an amplification effect, an asymptotic approximation for which in most cases statistical mechanics is invoked as a justification.

It is said that this picture conforms with Kant's warning that all we know concerns "things as appearances" not "things as such." A phenomenon is an "appearance." One may further note the similarity of Bohr's insistence on a description of $M$ in terms of classical physics with Kant's understanding of space, time and causality as a priori categories of the mind. This allows to speak about "objective things," "things for all" (at least for all physicists) instead of the fictitious "things as such." While this may suggest that the concept of "things as such" is superfluous because we can never know anything about them anyway it is not imperative to follow this suggestion. Physics has lived on the hypothesis that there are "things as such," real things of which we observe shadows, the phenomena and it has tried to infer the nature and properties of the hypothetical real things from the phenomena. While the latter cannot tell us what the real things are they tell us with a vengeance what they cannot be. With the increasing body of experimental information about phenomena and their interrelation it becomes less and less easy to devise a model of real things which is in harmony with the observations. Indeed the problem faced in the development of quantum theory has not been the ambiguity inherent in the attempt to infer properties of real things from the phenomena but the inability of devising any coherent realistic picture conforming with the observed phenomena.

A prime example is the notion of a particle, say an electron. Under suitable conditions it can be isolated in some macroscopic space-time region and considered as a physical system $\Sigma$. One does not doubt the reality of its existence. But one finds that one cannot assume that it, the individual electron, has a real trajectory in spacetime (a world line). The statement is not only that we do not know (or cannot know) the position at a given time due to the uncertainty principle but that one would be led to a host of paradoxa if one tried to assume that the electron has "really" some position. A particle does not mark a point in space at any given time. Usually one adds "unless it is measured." The measurement process involves the interaction of the electron with another particle, say a molecule in a screen or photographic plate. This marks (approximately) a point in space-time (not in space at arbitrary time). It 
is tempting to abstract from this the picture that there exist real, individual events and that a point (or rather a region) in space-time is an attribute of an event whereas we should not consider a point in space at a given time as an attribute of a particle. In other words there exist marked points in space-time but they are not connected by marked world lines. One may note, incidentally, that this fits well with relativity theory which uses the word "event" in the sense of a marked point in space-time as the most elementary concept.

We must ask now whether there is any evidence forbidding us to assume that an event is real unless it is macroscopically amplified or recorded in consciousness. The essential point here is the irreversibility and, related to it, the separability of events. It is traditional in theoretical physics to associate irreversibility with statistical mechanics. The fundamental laws are supposed to be time reflection invariant and the tendency towards equilibrium in the future rather than in the past is explained by "coarse graining" together with some assumptions about the initial conditions. On the scale of laboratory experiments the latter assumption can be related to the "psychological arrow of time" basic to the mind. The initial conditions are set when the experimenter comes to the laboratory and creates order (or havoc). On the large scale the initial conditions must be taken as an input from cosmology and the continuity of the arrow of time throughout the universe may be credited to the big bang. I want to suggest here that - once one accepts indeterminism - there is no reason against including irreversibility as part of the fundamental laws of nature. This is done if one accepts the notion of a real event as one fundamental concept. The past events and their causal ties may be subsumed under the notion of "state" which gives the potentiality for the birth of new events. The realization of a specific pattern of new events, the selection of facts from possibilities is spontaneous and apart from conservation laws - it is governed by probability. It should be stressed that this picture does not touch CTP-invariance or detailed balancing. As Lüders [1] has pointed out the term "time reversal" should be repaced by "motion reversal." One does not change the arrow of time but compares states with mirrored velocities.

Can the point of view described above be maintained in more general situations? Consider a typical collision experiment, a beam of particles bombarding a material target. Depending on the energy and energy transfer this may be idealized as a collision of the beam particle with different reaction partners. At low energies the partner is a molecule or even larger structure, for higher energies the molecular binding becomes irrelevant and we may consider a nucleus or electron as the reaction partner, then a nucleon, then perhaps a "constituent quark." Events in the sense of the experimentalist, namely a star of tracks in a photographic emulsion or bubble chamber emerge more and more conspicuously. Such a phenomenon has a center, the apex of the star, the dominant event in our sense. Its position in spacetime is sharp as compared to the range of locations allowed by the preparation of the beam. In other words the individual event marks one point from a wide range of choices. Of course we could not know about this marked point without the presence of photographic emulsion or some other detecting device by means of which a recorded phenomenon results via the secondary ionization events caused by the reaction products. Yet the natural explanation seems that we are dealing with the spontaneous birth of a fact from possibilities among which the nonoccurrence of an event figures also with high probability. By becoming a fact the event is irreversible 
and this irreversibility does not ask for an explanation in terms of statistical mechanics or the consciousness of an observer.

\section{History in Terms of Events and Causal Ties}

The preceding discussion suggests that the following concepts may be appropriate for an idealized description of the evolving history of the physical world. We have objects, called events, and arrows, called causal ties. An arrow leads from one event, its source, to another event, its target. The events are partially ordered. Event $\beta$ is called later than $\alpha$ if it is the target of a sequence of arrows of which the first has its source in $\alpha$. Mathematically the structure could be called a category with a boundary. The boundary is formed by all events which are sources of unsaturated arrows, arrows which have not (yet) found a target. The structure is evolving by the formation of new events absorbing unsaturated arrows so that events which were on the boundary now become inner. A cut $S$ through the arrows dividing the events into two sets $F_{S}$ and $P_{S}$ (the future and the past with respect to $S$ ) such that no event in $P_{S}$ is later than any event in $F_{S}$ and that $P_{S}$ contains no boundary points is the analogue of a space-like surface in classical space-time.

To transform this picture into a theory it is necessary

(i) to endow the set of possible events and possible arrows with further mathematical structure,

(ii) to state the restrictions in connecting events by arrows,

(iii) to give the scheme by which the probability for the appearance of a specific pattern (subset of events and arrows) may be computed from information about the past history,

(iv) to establish the relation to an observer and his instruments, essentially to classical space-time.

I am not able to present such a theory here. What we can do is to compare the picture to quantum field theory in the regime of low density and conspicuous events. In this regime causal ties correspond to particles ${ }^{1}$ and events to collision processes between particles. If, in quantum field theory, we describe an overall process where initially $n$ particles are present with centers of mass $x_{i}(i=1, \ldots, n)$ (defined by preparation or knowledge of previous events) and at a much later time the reaction products are detected in the neighborhood of the points $y_{k}(k=1, \ldots, m)$, then the probability amplitude may be written as

$$
A=\sum A_{G},
$$

where to each $A_{G}$ corresponds a graph consisting of the external vertices $x_{i}, y_{k}$, some internal vertices $z_{l}(l=1, \ldots, r)$ and lines connecting vertices (at most one line between two vertices). A typical graph with $n=3, m=4, r=4$ is drawn in Fig. 1. A magnified picture of one vertex $z_{l}$ is drawn in Fig. 2. We mark, for each line attached to it a point $z_{l}^{\alpha}(\alpha=1, \ldots, f)$, where $f$ is the number of lines meeting at the vertex. The vertex is then represented by a function $\Gamma\left(z_{l}^{1}, \ldots, z_{l}^{f}\right)$ which depends only on the

\footnotetext{
${ }^{1}$ Not necessarily elementary particles. They may be atoms, molecules...
} 
differences $z_{l}^{\alpha}-z_{l}^{\beta}$ and has short extension in these difference vectors. The line beginning at the point $z_{l}^{\alpha}$ and ending at $z_{l^{\prime}}^{\alpha^{\prime}}$ is represented by a propagator $\Delta\left(z_{l}^{\alpha}-z_{l^{\prime}}^{\alpha^{\prime}}\right)$. The contribution $A_{G}$ is the integral over the $z_{l}^{\alpha}$ of the product of the vertex functions and propagators. The decomposition (1) results in quantum field theory by a decomposition of time-ordered functions into shortly extended vertex parts and long extended parts along the lines indicated by Symanzik's structure analysis $[2]^{2}$. Next one notes that for large (time-like) distances between the vertices in Fig. 1 the propagators may be replaced by $\Delta_{+}$-functions of particles with a definite mass.
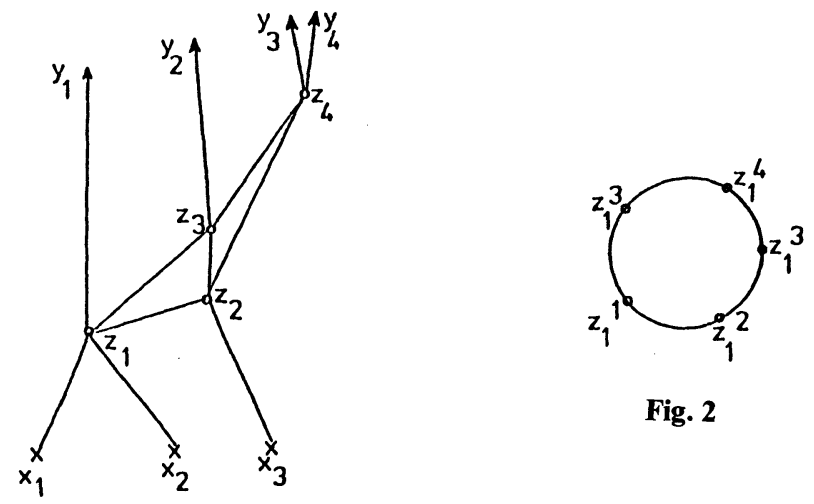

Fig. 2

Fig. 1

Expressing the functions $\Gamma$ and $\Delta$ by their Fourier decomposition and evaluating the integral for $A_{G}$ by the method of stationary phase, which is good if the distances between the points $x_{i}, y_{k}, z_{l}$ are large compared to the extension of the $\Gamma$-functions, one finds for each line a relevant momentum $p$ which is classically related to the end points, say $z_{l}, z_{l}$, by

$$
p=m\left(z_{l}-z_{l^{\prime}}\right)\left(\left(z_{l}-z_{l^{\prime}}\right)^{2}\right)^{-1 / 2},
$$

where $m$ is the mass value to which the line belongs and $z_{l}, z_{l}$, are some mean values of the $z_{l}^{\alpha}, z_{l^{\prime}}^{\alpha^{\prime}}$. We have furthermore momentum conservation at each vertex which gives $4 r$ equations for the $r$ unknown points $z_{l}$. Thus the space-time positions of the internal vertices are determined from the diagram for $A_{G}$ in terms of the external points $x_{i}, y_{k}$ and the mass values of the particles.

The probability $|A|^{2}$ for the overall process decomposes into

$$
|A|^{2}=\sum\left|A_{G}\right|^{2}
$$

if the distances between the external vertices are large because the interference terms are then negligable as different graphs give widely different configurations of internal vertices. Finally one sees that if we insert an initial space-like plane and the

\footnotetext{
${ }^{2}$ If the particle is "composite" then the point $z_{l}^{\alpha}$ corresponds to the center of mass and $\Gamma$ is obtained by a convolution of internal wave functions and ordinary vertex functions. Instable particles with a life time large compared to the extension of vertex functions can be treated as stable by adding decay vertices
} 
intersections of the wave functions of the initial particles with this plane are clearly separated (corresponding to the "low density" assumption) then the internal vertices will also be well apart and only a small number of graphs can contribute.

Summing up: in the low density case the multiple collision formalism of quantum field theory agrees with the picture that the total statistics of a process beginning with points $x_{i}$ and ending with points $y_{k}$ is given by the sum of probabilities for individual histories corresponding to graphs described at the beginning of this section and such that each intermediate event has a definite mean position in space-time with a short intrinsic extension.

The most severe challenge to any concept of reality in quantum physics comes from the discussion originating in the Einstein-Podolsky-Rosen argument. While in its original form, dealing with momentum and position measurements, the effect is wiped out in the long distance regime due to the spreading of wave packets; its adaptation to discrete quantum numbers (spin) persists. The (theoretically) simplest case is the decay of a spinless particle into two particles of spin $\frac{1}{2}$ and subsequent Stern-Gerlach experiments deciding whether the spin orientation is up or down with respect to chosen directions $e_{1}, e_{2}$ of the Stern-Gerlach magnets. There are 4 possible outcomes of an overall experiment which may be denoted by,+++- , ,-+-- and, correspondingly 4 probabilities $p_{i k}(i, k=+,-)$ depending on the directions $e_{1}, e_{2}$. If a "naive" concept of reality (underlying the original proposal of Einstein-Podolsky-Rosen) were tenable these probabilities should satisfy an inequality derived by Bell. The quantum mechanical predictions violate this and actual experiments performed decide against Bell's inequality and in favour of quantum mechanics. The important message is that - due to conservation of angular momentum - there are correlations in the probability matrix $p_{i k}$ which persist irrespective of the distance of the Stern-Gerlach events in space-time. From the point of view proposed at the beginning of this section this concerns item (iii) in the list of demands on the theory. It tells that the probability of a single event, considering only its backward causal ties, is not a meaningful concept. One must consider probabilities of patterns and these will not, in general, factorize. This is related to the fact that if we take a boundary in the evolving category of events and ties then there is no set of alternatives for a "next" event. Many subsequent patterns are possible and a set of alternatives (needed for the concept of probability) results only when all ties emanating from the boundary events become saturated.

If one goes beyond the low density regime one may take either one of two attitudes. One may argue that the concept of a history formed by events and causal ties is an approximation, suitable only in special circumstances. Or one may maintain that the transformation of possibilities into facts must be an essential ingredient which must be included in the fundamental formulation of the theory. C. F. v. Weizsäcker has emphasized for many years that the use of probabilities in statistical mechanics and quantum theory is necessarily always forward directed since the past is factual and the future open [3]. If irreversibility is introduced on a fundamental level as proposed above then the coincidence of the different "arrows of time" (psychological, thermodynamic, cosmological...) [4] is immediate and, in particular dissociated from any cosmological model. An early attempt to derive 4-dimensional space-time from assumptions about ties between events has been made by D. Finkelstein [5]. The above comparison with quantum field theory indicates 
that classical space-time is related to event structures by asymptotic approximations and the two attitudes mentioned differ by choosing classical space time or event structures as the primary concepts of the theory. It seems clear, however, that the marking of a point in space-time can only be realized physically by an event. Empty space-time, i.e. space-time without events, is probably a meaningless concept.

\section{References}

1. Lüders, G.: Dan. Mat. Fys. Medd. 28, 1195 (1954)

2. Symanzik, K.: J. Math. Phys. 1, 249 (1960)

3. Weizsäcker, C.F. v.: Probability and quantum mechanics. Br. J. Phil. Sci. 24, 321 (1973) Aufbau der Physik. München: dtv 1988

4. Zeh, H.D.: Die Physik der Zeitrichtung. Lecture Notes in Physics. Berlin, Heidelberg, New York: Springer 1984

5. Finkelstein, D.: Space-time code. Phys. Rev. 184, 1261 (1968)

Communicated by A. Jaffe

Received April 13, 1990 
